\title{
Standard paths in another composition poset
}

\author{
Jan Snellman \\ Department of Mathematics, Stockholm University \\ SE-10691 Stockholm, Sweden \\ Jan.Snellman@math.su.se
}

Submitted: Oct 8, 2003; Accepted: Oct 17, 2004; Published: Oct 26, 2004

\begin{abstract}
Bergeron, Bousquet-Mélou and Dulucq [1] enumerated paths in the Hasse diagram of the following poset: the underlying set is that of all compositions, and a composition $\mu$ covers another composition $\lambda$ if $\mu$ can be obtained from $\lambda$ by adding 1 to one of the parts of $\lambda$, or by inserting a part of size 1 into $\lambda$.

We employ the methods they developed in order to study the same problem for the following poset, which is of interest because of its relation to non-commutative term orders : the underlying set is the same, but $\mu$ covers $\lambda$ if $\mu$ can be obtained from $\lambda$ by adding 1 to one of the parts of $\lambda$, or by inserting a part of size 1 at the left or at the right of $\lambda$. We calculate generating functions for standard paths of fixed width and for standard paths of height $\leq 2$.
\end{abstract}

\section{Definition of standard paths}

By a composition $P$ we mean a sequence of positive integers $\left(p_{1}, p_{2}, \ldots, p_{k}\right)$, which are the parts of $P$. We define the length $\ell(P)$ of $P$ as the number of parts, and the weight $|P|=\sum_{i=1}^{k} p_{k}$ as the sum of its parts. If $P$ has weight $n$ then $P$ is a composition of $n$, and we write $P \vDash n$.

We say that a composition $Q$ covers a composition $P$ if $Q$ is obtained from $P$ either by adding 1 to a part of $P$, or by inserting a part of size 1 to the left, or by inserting a part of size 1 to the right. Thus, $P=\left(p_{1}, p_{2}, \ldots, p_{k}\right)$ is covered by

1. $\left(1, p_{1}, \ldots, p_{k}\right)$,

2. $\left(p_{1}, \ldots, p_{k}, 1\right)$,

3. and, for $1 \leq i \leq k,\left(p_{1}, \ldots, p_{i}+1, \ldots, p_{k}\right)$.

Extending this relation by transitivity makes the set of all compositions into a partially ordered set, which we denote by $\mathfrak{N}$. This is in accordance with the notations in the author's 
article A poset classifying non-commutative term orders [3], where $\mathfrak{N}$ was used for the following isomorphic poset of words: the underlying set is $X^{*}$, the free associative monoid on $X=\left\{x_{1}, x_{2}, x_{3}, \ldots\right\}$, and $m_{1}=x_{i_{1}} \cdots x_{i_{r}}$ is smaller than $m_{2}$ if $m_{2}$ can be obtained from $m_{1}$ by a sequence of operations of the form

(i) Multiply by a word to the left,

(ii) Multiply by a word to the right,

(iii) Replace an occurring $x_{i}$ with an $x_{j}$, with $j>i$.

The bijection $\left(p_{1}, p_{2}, \ldots, p_{k}\right) \mapsto x_{p_{1}} \cdots x_{p_{k}}$ is an order isomorphism between these two partially ordered sets.

On the other hand, the partial order $\Gamma$ on compositions studied by Bergeron, BousquetMélou and Dulucq in Standard paths in the composition poset [1] is different, since in $\Gamma$ the composition $P=\left(p_{1}, p_{2}, \ldots, p_{k}\right)$ is covered by

1. $\left(1, p_{1}, \ldots, p_{k}\right)$,

2. $\left(p_{1}, \ldots, p_{k}, 1\right)$,

3. for $1 \leq i \leq k,\left(p_{1}, \ldots, p_{i}+1, \ldots, p_{k}\right)$,

4. for $1 \leq i<k,\left(p_{1}, \ldots, p_{i}, 1, p_{i+1}, \ldots, p_{k}\right)$.

$\Gamma$ and $\mathfrak{N}$ coincide for compositions of weight $\leq 4$. In Figure 1 this part of the Hasse diagram is depicted. We have that $(2,2) \leq(2,1,2)$ in $\Gamma$ but not in $\mathfrak{N}$, so the rest of the respective Hasse diagrams differ.

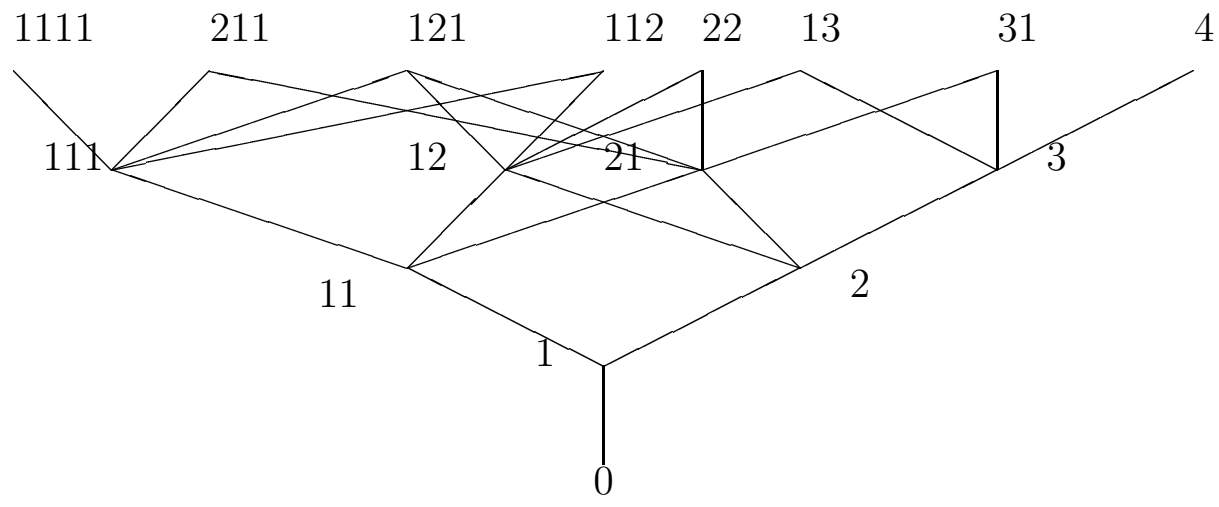

Figure 1: The Hasse diagram of $\mathfrak{N}$.

Following [1] we define a standard path of length $n$ to be a sequence $\gamma=\left(P_{0}, P_{1}, P_{2}, \ldots, P_{n}\right)$ of compositions such that

$$
P_{0} \prec P_{1} \prec P_{2} \prec \cdots \prec P_{n}, \quad P_{i} \vDash i .
$$


The partial order is now that of $\mathfrak{N}$. For instance,

$$
\rho=((),(1),(1,1),(1,2),(1,1,2))
$$

is a standard path of length 4, corresponding to a saturated chain in Hasse diagram of $\mathfrak{N}$ between the minimal element () and the element $(1,1,2)$.

We furthermore define the diagram of a composition $P=\left(p_{1}, \ldots, p_{k}\right)$ to be the set of points $(i, j) \in \mathbb{Z}^{2}$ with $1 \leq j \leq p_{i}$. Alternatively, we can replace the node $(i, j)$ by the square with corners $(i-1, j-1),(i-1, j),(i, j-1)$ and $(i, j)$. So the composition

\section{$(1,1,2)$ has diagram}

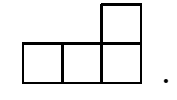

For a standard path $\gamma=\left(P_{1}, \ldots, P_{n}\right)$ ending at $P_{n}$ we label the boxes in the diagram of $P_{n}$ in the order that they appear in the path. To avoid ambiguity, we use the convention that whenever $P_{i}$ consists of $i$ ones and $P_{i+1}$ consists of $i+1$ ones, the extra one is considered to have been added to the left. So for the path $\rho$

the corresponding tableau is $4 / 2 \mid 1$.

Clearly, two different standard paths give rise to different tableau. Furthermore, the tableau that occurs as tableau of standard paths must be increasing in every column, and have the additional property that whenever the numbers $1,2, \ldots, k$ occur as a contiguous sequence on the bottom row, then that sequence is $k, k-1, \ldots, 2,1$. This is a necessary but not sufficient condition.

The underlying diagram of a tableau is called its shape, and we define the shape of a standard path to be the shape of its tableau. We define the height and width of a diagram to be the height and width of the smallest rectangle containing it. Hence, the standard path $\rho$ has width 2 and height 1 .

\section{Enumeration of standard paths of fixed width}

Let $\mathfrak{N}_{(k)}$ denote the subposet of compositions of width $k$. For a path $\gamma$ of shape $\left(p_{1}, p_{2}, \ldots, p_{k}\right)$ we set

$$
v(\gamma)=x_{1}^{p_{1}} x_{2}^{p_{2}} \cdots x_{k}^{p_{k}}
$$

We want to compute the generating function

$$
f_{k}\left(x_{1}, \ldots, x_{k}\right)=\sum_{\gamma \text { path of width } k} v(\gamma)
$$

Theorem 1. The generating function $f_{k}\left(x_{1}, \ldots, x_{k}\right)$ of standard paths of width $k$ is a rational function given by the following recursive relation: $f_{0}=1, f_{1}\left(x_{1}\right)=x_{1}\left(1-x_{1}\right)^{-1}$, and for $k>1$

$$
f_{k}\left(x_{1}, \ldots, f_{k}\right)=\frac{x_{1} f_{k-1}\left(x_{2}, \ldots, x_{k}\right)+x_{k} f_{k-1}\left(x_{1}, \ldots, x_{k-1}\right)-x_{1} \cdots x_{k}}{1-x_{1}-\ldots-x_{k}}
$$

Proof. A tableau of width $k$ can be obtained by adding a new cell either 
- at the top of a column of another tableau of width $k$,

- at the beginning of a tableau of width $k-1$,

- or at the end of a tableau of width $k-1$.

These three cases correspond respectively to $\left(x_{1}+x_{2}+\ldots+x_{k}\right) f_{k}$, to $x_{1} f_{k-1}\left(x_{2}, \ldots, x_{k}\right)$, and to $x_{k} f_{k-1}\left(x_{1}, \ldots, x_{k-1}\right)$. However, if the tableau has shape $(1, \ldots, 1)$ then the last two operations give the same result. Hence

$$
f_{k}=\left(x_{1}+x_{2}+\ldots+x_{k}\right) f_{k}+x_{1} f_{k-1}\left(x_{2}, \ldots, x_{k}\right)+x_{k} f_{k-1}\left(x_{1}, \ldots, x_{k-1}\right)-x_{1} \cdots x_{k},
$$

from which (51) follows.

We obtain successively

$$
\begin{aligned}
& f_{0}=1 \\
& f_{1}=\frac{x_{1}}{1-x_{1}} \\
& f_{2}=\frac{x_{1} x_{2}\left(1-x_{1} x_{2}\right.}{\left(1-x_{1}\right)\left(1-x_{2}\right)\left(1-x_{1}-x_{2}\right)} \\
& f_{3}=\left(x_{1}{ }^{2} x_{2}{ }^{2} x_{3}+x_{1}{ }^{2} x_{2} x_{3}{ }^{2}+x_{1} x_{2}{ }^{3} x_{3}+x_{1} x_{2}{ }^{2} x_{3}{ }^{2}-x_{1}{ }^{2} x_{2}{ }^{2}\right. \\
&-4 x_{1}{ }^{2} x_{2} x_{3}-x_{1}{ }^{2} x_{3}{ }^{2}-x_{1} x_{2}{ }^{3}-7 x_{1} x_{2}{ }^{2} x_{3}-4 x_{1} x_{2} x_{3}{ }^{2}- \\
& x_{2}{ }^{3} x_{3}-x_{2}{ }^{2} x_{3}{ }^{2}+2 x_{1}{ }^{2} x_{2}+2 x_{1}{ }^{2} x_{3}+5 x_{1} x_{2}{ }^{2}+12 x_{1} x_{2} x_{3} \\
&+ 2 x_{1} x_{3}{ }^{2}+x_{2}{ }^{3}+5 x_{2}{ }^{2} x_{3}+2 x_{2} x_{3}{ }^{2}-5 x_{1} x_{2}-4 x_{1} x_{3} \\
&\left.-3 x_{2}{ }^{2}-5 x_{2} x_{3}+x_{2}+1\right) \\
& \times x_{1} x_{2} x_{3} \\
& \times\left(1-x_{1}\right)^{-1}\left(1-x_{2}\right)^{-1}\left(1-x_{3}\right)^{-1}\left(1-x_{1}-x_{2}\right)^{-1}\left(1-x_{2}-x_{3}\right)^{-1} \\
& \times\left(1-x_{1}-x_{2}-x_{3}\right)^{-1}
\end{aligned}
$$

Theorem 2. For each $k$,

$$
f_{k}\left(x_{1}, \ldots, x_{k}\right)=\frac{x_{1} \cdots x_{k}}{\prod_{i=1}^{k} \prod_{j=i}^{k}\left(1-x_{i}-x_{i+1}-\ldots-x_{j}\right)} \tilde{f}_{k}\left(x_{1}, \ldots, x_{k}\right)
$$

where $\tilde{f}_{k}$ is a polynomial.

Proof. This is true for $k=0,1$. Assume that $f_{k-1}$ has the above form. Then

$$
\begin{gathered}
f_{k}\left(1-x_{1}-\cdots-x_{k}\right)=x_{1} f_{k-1}\left(x_{2}, \ldots, x_{k}\right)+x_{k} f_{k-1}\left(x_{1}, \ldots, x_{k-1}\right)-x_{1} \cdots x_{k} \\
=x_{1} x_{2} \cdots x_{k} \tilde{f}_{k-1}\left(x_{2}, \ldots, x_{k}\right) \prod_{i=2}^{k} \prod_{j=i}^{k}\left(1-x_{i}-\cdots-x_{j}\right)^{-1} \\
+x_{k} x_{1} \cdots x_{k-1} \tilde{f}_{k-1}\left(x_{1}, \ldots, x_{k-1}\right) \prod_{i=1}^{k-1} \prod_{j=i}^{k-1}\left(1-x_{i}-\cdots-x_{j}\right)^{-1}-x_{1} \cdots x_{k}
\end{gathered}
$$


hence

$$
\begin{aligned}
& \frac{f_{k}\left(1-x_{1}-\cdots-x_{k}\right) \prod_{i=1}^{k} \prod_{j=i}^{k}\left(1-x_{i}-\cdots-x_{j}\right)}{x_{1} \cdots x_{k}} \\
& =\tilde{f}_{k-1}\left(x_{2}, \ldots, x_{k}\right) \prod_{j=1}^{k}\left(1-x_{1}-\cdots-x_{j}\right)+\tilde{f}_{k-1}\left(x_{1}, \ldots, x_{k-1}\right) \prod_{i=1}^{k}\left(1-x_{i}-\cdots-x_{k}\right) \\
& -\prod_{i=1}^{k} \prod_{j=i}^{k}\left(1-x_{i}-\cdots-x_{j}\right)
\end{aligned}
$$

Let $a_{n, k}$ denote the number of standard paths of width $k$ and length $n$, and let

$$
L_{k}(t)=\sum_{n \geq 0} a_{n, k} t^{n}
$$

be the generating function for the number of standard paths of width $k$ and length $n$. Then $L_{k}(t)=f_{k}(t, \ldots, t)$. This substitution results in some cancellation in the numerator and denominator; we have that

$$
\begin{aligned}
& L_{1}(t)=\frac{t}{1-t} \\
& L_{2}(t)=\frac{t^{2}(1+t)}{(1-t)(1-2 t)} \\
& L_{3}(t)=\frac{t^{3}\left(1+5 t-2 t^{2}\right)}{(1-t)(1-2 t)(1-3 t)} \\
& L_{4}(t)=\frac{t^{4}\left(1+16 t-15 t^{2}+6 t^{3}\right)}{(1-t)(1-2 t)(1-3 t)(1-4 t)} \\
& L_{5}(t)=\frac{t^{5}\left(1+42 t-65 t^{2}+62 t^{3}-24 t^{4}\right)}{(1-t)(1-2 t)(1-3 t)(1-4 t)(1-5 t)}
\end{aligned}
$$

\section{Proposition 3.}

$$
L_{k}(t)=\frac{t^{k} \tilde{L}_{k}(t)}{\prod_{i=1}^{k}(1-i t)}
$$

where $\tilde{L}_{k}(t)$ is a polynomial of degree $k-1$ with $\tilde{L}_{k}(1)=2^{k-1}$.

Proof. The recursive relation (5) specializes to

$$
L_{k}=\frac{2 t L_{k-1}-t^{k}}{1-k t}
$$

Assume (11) for a fixed $k$; then (12) gives

$$
L_{k+1}=\frac{2 t^{k+1} \tilde{L}_{k}-t^{k+1} \prod_{i=1}^{k}(1-i t)}{\prod_{i=1}^{k+1}(1-i t)}
$$


Since $\tilde{L_{k}}$ has degree $k-1$ and evaluates to $2^{k-1}$ at 1 , we get that $\tilde{L}_{k+1}=2 \tilde{L}_{k}-\prod_{i=1}^{k}(1-i t)$ has degree $k$ and evaluates to $2^{k}$ at 1 . The assertion now follows by induction.

Corollary 4. For a fixed $k$,

$$
a_{n+k, k} \sim \frac{k^{k-1}}{(k-1) !} k^{n} \quad \text { as } n \rightarrow \infty
$$

Proof. This follows from the previous Proposition, and the partial fraction decomposition

$$
\begin{aligned}
\prod_{i=1}^{k}(1-i t)^{-1} & =\sum_{j=1}^{k} v_{j, k}(1-i t)^{-1} \\
v_{k, k} & =\frac{k^{k-1}}{(k-1) !}
\end{aligned}
$$

\section{Enumeration of standard paths of height at most two}

Let $\mathfrak{N}_{n, i, j}^{(k)}$ denote the poset of compositions of $n$ with height $\leq k$, having $i$ parts of size 1 and $j$ parts of size $\geq 2$. Let $\gamma_{n, i, j}^{(k)}$ be the number of standard paths with endpoint in $\mathfrak{N}_{n, i, j}^{(k)}$.

We will derive a recurrence relation for $\gamma_{n, i, j}^{(2)}$. Note that a tableau of height $\leq 2$, with $i$ parts of size 1 and $j$ parts of size 2 , has a total of $n=i+2 j$ boxes, so $\gamma_{n, i, j}^{(2)}=0$ unless $n=i+2 j$. Put $c_{i, j}^{(2)}=\gamma_{i+2 j, i, j}^{(2)}$. A tableau with $i$ parts of size 1 and $j$ parts of size 2 , can be obtained

- from a tableau with $i-1$ parts of size 1 and $j$ parts of size 2 , by adding a part of size 1 to the left,

- or from a tableau with $i-1$ parts of size 1 and $j$ parts of size 2 , by adding a part of size 1 to the right,

- or from a tableau with $i+1$ parts of size 1 and $j-1$ parts of size 2 , by adding a box to a part of size 1 .

For the composition consisting of $n$ ones the first two ways are identical, which gives the recurrence

$$
\begin{aligned}
\gamma_{n, i, j}^{(2)} & =2 \gamma_{n-1, i-1, j}^{(2)}+(i+1) \gamma_{n-1, i+1, j-1}^{(2)}-\delta_{j}^{0} \\
c_{i, j}^{(2)} & =2 c_{i-1, j}^{(2)}+(i+1) c_{i+1, j-1}^{(2)}-\delta_{j}^{0}
\end{aligned}
$$

where $\delta_{i}^{j}$ is the Kronecker delta. We get that $c_{n, 0}^{(2)}=\gamma_{n, n, 0}^{(2)}=1, \gamma_{n, i, 0}^{(2)}=0$ for $i \neq n$. For small values of $i, j, c_{i, j}^{(2)}$ is as in table 1 


\begin{tabular}{|r|rrrrrrrr|}
\hline & $\mathrm{j}$ & 0 & 1 & 2 & 3 & 4 & 5 & 6 \\
$\mathrm{i}$ & & & & & & & & 7 \\
\hline 0 & 1 & 1 & 4 & 30 & 336 & 5040 & 95040 & 2162160 \\
1 & 1 & 4 & 30 & 336 & 5040 & 95040 & 2162160 & 57657600 \\
2 & 1 & 11 & 138 & 2184 & 42480 & 986040 & 26666640 & 824503680 \\
3 & 1 & 26 & 504 & 10800 & 265320 & 7447440 & 236396160 & 8393898240 \\
4 & 1 & 57 & 1608 & 45090 & 1368840 & 45765720 & - & - \\
5 & 1 & 120 & 4698 & 167640 & 6174168 & 242686080 & - & - \\
6 & 1 & 247 & 12910 & 572748 & 25192440 & 1151011680 & - & - \\
7 & 1 & 502 & 33924 & 1834872 & 95091360 & 4999942080 & - & - \\
8 & 1 & 1013 & 86172 & 5588310 & 337239840 & - & - & - \\
\hline
\end{tabular}

Table 1: Values of $c_{i, j}^{(2)}$ for small $i, j$

Theorem 5. Put

$$
P_{k}(x)=\sum_{n=0}^{\infty} c_{n, k}^{(2)} x^{n}
$$

Then $P_{0}(x)=(1-x)^{-1}$ and

$$
P_{k}(x)=\frac{\frac{d}{d x} P_{k-1}(x)}{1-2 x}
$$

Proof. Since $c_{n, 0}^{(2)}=1$ it follows that $P_{0}(x)=\sum_{n=0}^{\infty} c_{n, 0}^{(2)} x^{n}=(1-x)^{-1}$.

Now, multiply (15) with $x^{i}$ and sum over all $i \geq 0$ to get that

$$
\sum_{i \geq 0} c_{i, j}^{(2)} x^{i}=2 \sum_{i \geq 1} c_{i-1, j}^{(2)} x^{i}+\sum_{i \geq 0}(i+1) c_{i+1, j-1}^{(2)} x^{i}
$$

which means that

$$
P_{j}(x)=2 x P_{j}(x)+P_{j-1}^{\prime}(x)
$$

We get that

$$
\begin{aligned}
& P_{1}(x)=(1-x)^{-2}(1-2 x)^{-1} \\
& P_{2}(x)=2 !(1-x)^{-3}(1-2 x)^{-3}(2-3 x) \\
& P_{3}(x)=3 !(1-x)^{-4}(1-2 x)^{-5}\left(5-14 x+10 x^{2} x\right) \\
& P_{4}(x)=4 !(1-x)^{-5}(1-2 x)^{-7}\left(14-56 x+76 x^{2}-35 x^{3}\right)
\end{aligned}
$$

and in general

$$
P_{k}(x)=k !(1-x)^{-1-k}(1-2 x)^{1-2 k} Q_{k}(x)
$$

where $Q_{k}(x)$ is a polynomial of degree $k-1$, with $Q_{k}(1)=(-1)^{k+1}$. 
Theorem 6. Put

$$
P(x, y)=\sum_{i, j \geq 0} c_{i, j}^{(2)} x^{i} \frac{y^{j}}{j !}
$$

Then

$$
P(x, y)=\frac{2}{1+\sqrt{1-4\left(y+x-x^{2}\right)}}
$$

Proof. We get from the recurrence relation (16) that

$$
(1-2 x) \frac{\partial P}{\partial y}=\frac{\partial P}{\partial x}
$$

Furthermore, $P_{0}(x)=P(x, 0)=(1-x)^{-1}$. The proposed $P(x, y)$ satisfies (24) and the initial condition, so it is the solution.

Theorem 7. With the notations above,

$$
\begin{aligned}
& c_{0, n}^{(2)}=\frac{(2 n) !}{(n+1) !} \\
& c_{1, n}^{(2)}=c_{0, n+1}^{(2)}=\frac{(2(n+1)) !}{(n+2) !} \\
& c_{2, n}^{(2)}=\frac{1}{2} c_{0, n+2}^{(2)}-c_{0, n+1}^{(2)}=\frac{1}{16} \frac{\left(2 n^{2}+6 n+3\right) 2^{2 n+6} \Gamma(n+3 / 2)}{(n+3) \sqrt{\pi}(n+2)}
\end{aligned}
$$

Thus, the sequences $\left(c_{0, n}^{(2)}\right)_{n=0}^{\infty}$ and $\left(c_{1, n}^{(2)}\right)_{n=0}^{\infty}$ are translations of the sequence A001761 in The On-Line Encyclopedia of Integer Sequences 2] (OEIS).

Proof. We have that

$$
P(0, y)=\frac{2}{1+\sqrt{1-4 y}}
$$

which is the well-known ordinary generating function for the Catalan numbers. This proves the formula for $c_{0, n}^{(2)}$. The recurrence (15) gives $c_{1, n}^{(2)}=c_{0, n+1}^{(2)}$ and $c_{2, n}^{(2)}=\frac{1}{2} c_{0, n+2}^{(2)}-$ $c_{0, n+1}^{(2)}$. Combining these two results, and simplifying, yields the theorem.

\section{References}

[1] François Bergeron, Mireille Bousquet-Mélou, and Serge Dulucq. Standard paths in the composition poset. Ann. Sci. Math. Québec, 19(2):139-151, 1995.

[2] Neil J. A. Sloane. The on-line encyclopedia of integer sequences. http://www.research.att.com/ njas/sequences/index.html.

[3] Jan Snellman. A poset classifying non-commutative term orders. In Discrete models: Combinatorics, Computation, and Geometry, Discrete Mathematics and Theoretical Computer Science Proceedings AA (DM-CCG), pages 301-314, 2001. 